\title{
Improving old tricks: FX interpolation beyond alias for irregularly sampled data, for data with large gaps and for data with spatially variant dips
}

\author{
Mostafa Naghizadeh and Mauricio Sacchi (University of Alberta)
}

\section{SUMMARY}

For almost two decades, FX prediction filtering (Spitz, 1991) and FK interpolation (Gülünay, 2003) have provided a robust route to interpolate aliased seismic data. These are probably the only two interpolation methods that were built on sound and solid arguments for the interpolation of aliased events. They both have precise assumptions that one must honor to optimally interpolate seismic data. In essence, the data must be composed of a finite superposition of events with linear moveout. The latter is often validated by windowing the data. In addition, both methods require regularly sampled data and a good SNR in the low frequency portion of the spectrum where the vital non-aliased Fourier coefficients reside.

In this presentation we provide a series of improvements to FX prediction filtering. First, we show how one can use FX prediction filtering to interpolate irregularly sampled data. Multistep autoregressive reconstruction (Naghizadeh and Sacchi, 2007) uses regularized low frequency data components to estimate an ensemble of prediction filters that can be used to regularize aliased data components. MSAR is an extension of FX prediction filtering for the case of irregularly sampled data and/or data with gaps.

A multi-dimensional version of MSAR (Naghizadeh and Sacchi, 2009b) was also provided and applied to real data. Windowing is often required to validate the linear event assumption. FX prediction interpolation can be implemented in a recursive fashion that does not require windowing (Naghizadeh and Sacchi, 2009a). The recursive implementation of FX prediction interpolation permits to interpolate aliased data with spatially variant dips.

Finally, we show that the amplitude spectrum of prediction filters can also be used to define regions of spectral support in the wave number domain (Naghizadeh and Sacchi, 2009c). The latter leads to an FX prediction filter algorithm very similar to the Anti-leakege Fourier Transform proposed by (Xu et al. 2005).

\section{References}

Gülünay N, 2003, Seismic trace interpolation in the Fourier transform domain, Geophysics, 68, 1, 355-369

Naghizadeh M and M D Sacchi, 2007, Multistep autoregressive reconstruction of seismic records, Geophysics, 72, 6, V111-V118.

Naghizadeh M and M D Sacchi, 2009a, f-x adaptive seismic-trace interpolation, Geophysics, 74, 1, V9V16.

Naghizadeh M and M D Sacchi, 2009b, Robust reconstruction of aliased data using autrregressive spectral estimates, submitted to the EAGE 2009 conference. 
Naghizadeh M and M D Sacchi, 2009c, Seismic data reconstruction using multidimensional prediction filters, Geophysics Prospecting, accepted for publication

Spitz S., 1991, Seismic trace interpolation in the FX domain, Geophysics, 56, 6, 785-794

Xu S., Z. Yu, D. Pham, and G Lambare, 2005, Antileakage Fourier transform for seismic data regularization, Geophysics, 70, 4, V87-95. 